\title{
Europa: Zeitalter der Krisen
}

Fast zehn Jahre nach dem scheinbar plötzlichen Ausbruch der internationalen Finanzkrise stellt sich die Frage, wie stabil und zukunftsfähig der deutsche Finanzsektor ist. Dabei fallen als erstes die Schlagzeilen über die DAX-Banken auf. Diese Banken sind nach wie vor nicht zu ihrer alten Blüte zurückgekehrt. Noch stärker hat es etliche Landesbanken erwischt; gerade im Landesbankensektor sind starke „Konsolidierungsbemühungen“ feststellbar.

Eine Rückkehr in die „gute“ alte Zeit ist so oder so nicht möglich. Denn die Geschäftsmodelle der Vergangenheit standen auf tönernen Füßen. Die Idee war einfach: Es wurde auf Risikoprämien bei der Kreditvergabe gesetzt. Die mit dem Bankgeschäft verbundenen Risiken wurden mittels Annahmen und Algorithmen kleingerechnet. Die Prämien wurden hoch angesetzt. Unter diesen Umständen sollten dann nennenswerte Renditen aus den Finanzgeschäften entstehen. Dieses Geschäftsmodell ist vorbei. Die Rahmenbedingungen für das Kreditgeschäft wurden nach den Krisenerfahrungen auf der europäischen Ebene massiv verändert. Bankenregulierung und teilweise auch die Bankenaufsicht wurde zu einer wichtigen europäischen Gemeinschaftsaufgabe gemacht. Die Risiken werden unter den veränderten Rahmenbedingungen von der Regulierungsseite stärker eingepreist. Davon ist auch das deutsche Drei-Säulensystem, mit seinen Genossenschaftsbanken, dem öffentlich-rechtlichen Sektor und den privaten Geschäftsbanken betroffen. Herauskommen sollte ein Bankensektor, der sich erst gar nicht mehr dem Risiko eines Crashes ausliefert. Die neuen Regulierungen - insbesondere das sogenannte Basel-III-Paket - sollen langfristig stabilisierend wirken und somit dazu beitragen, dass eine Situation wie 2007/2008 nicht wieder entstehen kann. Das ist einerseits gut so, andererseits zeigt sich in der aktuellen Regulierung ein entscheidendes Dilemma der Politik: Es werden Lösungen für „alte“ Probleme geboten. Längst ist die Karawane weiter gezogen...

Da sind zum einen die immer stärker wachsenden Schattenbanken - Finanzierungsinstitute, die nur ausgewählte Bankdienstleistungen anbieten und sich wie die sogenannten FinTechs - die fortschreitende Digitalisierung zunutze machen und regulierungsarme Nischen suchen. Gerade die mit der Digitalisierung verbundenen technischen Möglichkeiten auch grenzüberschreitender Geldtransfers lassen die klassische Regulierung von Finanzdienstleistungen blass aussehen. Ein Smartphone ist in vielen Ländern schon Ersatz der traditionellen Bankfiliale. Hier entstehen Chancen, aber auch neue Gefahren. Die EU-Regulierung hinkt vollständig hinterher. Da sind zum anderen die Geschäftsbanken. Finanzinnovationen wie mobiles Banking sind in Deutschland nicht gerade ihre Stärke. Sie widmen sich zwar längst nicht mehr vorrangig der Finanzierung von Unternehmensinvestitionen, sondern - in Deutschland - vor allem der Wohnungsbaukreditierung. Dass die unspezifische Wohnungsbaufinanzierung leicht der Ausgangspunkt von Liquiditäts- und Solvenzkrisen im Bankensektor werden kann, ist bekannt. Bezogen auf den Bankensektor in Deutschland kann keine Entwarnung gegeben werden. Zwar liegen die Eigenkapitalrenditen im Durchschnitt wieder im positiven Bereich, doch das eigentliche Geschäftsfeld der Banken gerät zunehmend ins Wanken. Digitalisierungsgewinne lassen sich mit dem alten Modell schlecht abschöpfen. Finanzinnovation findet vielfach außerhalb des Bankensektors statt. Die Grenzen des alten Geschäftsmodells im digitalen Zeitalter zeigen sich auch daran, dass sich die Gewinne der Geschäftsbanken immer mehr aus Provisionen und immer weniger aus Zinsen speisen.

Kritiker meinen, das ist eine Folge der Niedrigzinspolitik der Zentralbank. Dem muss deutlich widersprochen werden. Banken leben bei der Kreditvergabe von der Differenz zwischen Soll- und Habenzinsen, also von der Differenz zwischen dem veranschlag-

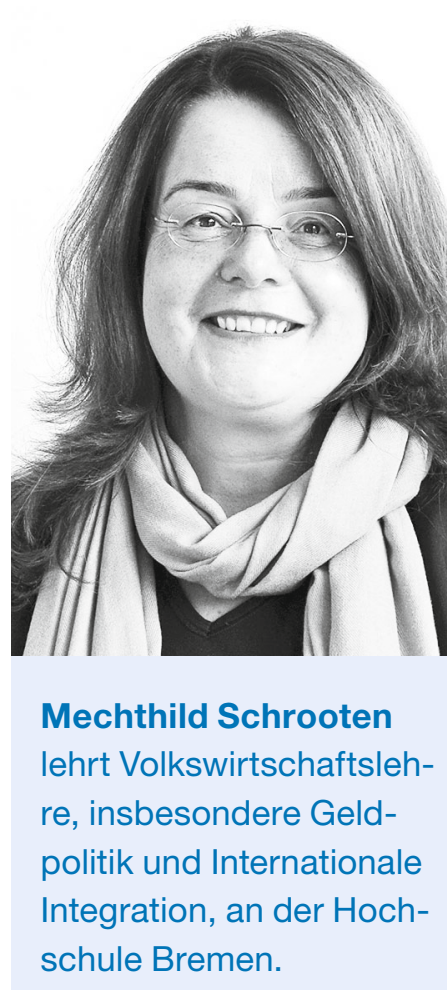

Mechthild Schrooten lehrt Volkswirtschaftslehre, insbesondere Geldpolitik und Internationale Integration, an der Hoch- 
ten Kreditzinssatz und dem Zinssatz, den die Banken zu ihrer eigenen Refinanzierung zahlen müssen. Niedrige Zinsen bedeuten daher erst einmal günstige Einkaufspreise für die Banken. Eigentlich müssten die Banken bei einer Nullzinspolitik der Zentralbank jubilieren. Tatsächlich sind die Refinanzierungskosten so gering wie nie zuvor. Das Zinsdilemma kommt aus einer anderen Ecke: Den Banken gehen die solventen Schuldner aus. In Zeiten geringer Einlagezinsen greifen die Unternehmen gern auf eigene Mittel zur Investitionsfinanzierung zurück. Die Staaten in der Europäischen Union sind mehr oder weniger an den Fiskalpakt gebunden. Die öffentlichen Haushalte fragen kaum noch zusätzliche Kredite nach - bestenfalls geht es um Umschichtungen von Schuldenbeständen. Im System ist eine merkliche Überliquidität. Geld sucht Anlagemöglichkeiten. Das drückt von sich aus das Zinsniveau. Diese Effekte lassen sich inzwischen auch an den Finanzierungsbedingungen der von der wirtschaftlichen Krise stark gebeutelten Euro- und EU-Staaten festmachen. Die Zeiten sind vorbei, in denen die Gläubigerseite Fantasiezinssätze für europäische Staatsanleihen ausrufen konnte. Dies ist das Verdienst der EZB: Sie hat gezeigt, wie Zusammenhalt funktionieren kann. Zusammenhalt organisiert Wettbewerb und Markt nach anderen Spielregeln. Das haben Konzerne längst erkannt.

Die Politik in Europa dagegen wirkt an vielen Stellen erkenntnisresistent. Die Gestaltung der EU wird oftmals dem Zufallsprinzip und Zufallsereignissen überlassen. Die EU reagiert einfach nur, in der aktiven Zukunftsgestaltung zeigt sie sich aber zögerlich. Die EU und auch die Eurozone haben vielen Wohlstand gebracht - heute wird das für die Mitgliedschaft in der Eurozone infrage gestellt. Tatsächlich gibt es viel zu viele Verlierer in den letzten Jahren. Nationalstaatliche und rechtspopulistische Tendenzen lassen sich allerdings nicht einseitig auf dieses Argument zurückführen. Gerade die mittel-osteuropäischen Staaten profitieren stark von der EU-Mitgliedschaft, setzen aber in vielen Fällen auf nationalistische Ideen.

In der Eurozone, aber auch in der gesamten EU wird immer klarer: Geld, das gemeinsame Geld und die gemeinsame Währung ist ein wichtiges Kommunikationsmittel. Und anders als es der Begriff der Eurokrise suggeriert, funktioniert das Kommunikationsmittel „Euro“ - zweifelsfrei nicht reibungslos, aber es funktioniert. Nach außen hat der Euro nie als internationale Leitwährung ernsthaft zur Debatte gestanden, selbst in den härtesten Krisenjahren. Das zeigt auch seine relative Wechselkursstabilität trotz aller europapolitischer Turbulenzen. Nach innen, also in der EU und der Eurozone, dagegen gibt es viele Diskussionen. Und das ist gut so. Denn endlich wird darüber gesprochen, was eine Währungsunion eigentlich bedeutet.

Letztendlich ist es klar, dass auch diese Krise der EU und der Eurozone in einer Vertiefung der Zusammenarbeit enden wird. Die Grundlagen dazu müssen jetzt hart erarbeitet werden. Es geht tatsächlich um europäische Werte: Ist es ein Wert an sich, Unternehmensrenditen zu garantieren, oder geht es um attraktive Lebensbedingungen von Menschen? Die EU und auch die Eurozone werden sich auf die Seite der Menschen stellen müssen und dürfen die Unternehmensgewinne trotzdem nicht aus den Augen verlieren. Das gemeinsame Geld, der Euro, kann dabei die Kommunikation erheblich erleichtern. Denn Wohlstand und auch die Verteilung von Wohlstand lassen sich in Geldeinheiten messen. Es gibt keinen Grund, die EU oder die Eurozone an der Verteilungsfrage scheitern zu lassen. Der Preis wäre viel zu hoch, die Folgen unkalkulierbar. Aber vielleicht geht es gar nicht allein um die Verteilungsfrage - vielleicht kommt das Brodeln aus dem Innern aus anderen Quellen. Identitätssuche? Auch hier kann das Kommunikationsmittel Euro helfen. Eine nationale Währung stellt gerade für Krisen-

Mechthild Schrooten

Hochschule Bremen

Mechthild.Schrooten@hs-bremen.de oder Schuldnerstaaten keine Problemlösung dar. Die Wettbewerbsnachteile würden in anderen Geldeinheiten gemessen; die Transaktionskosten würden erheblich steigen. Im klug organisierten Zusammenhalt liegt der Schlüssel zur Krisenbewältigung. 